\title{
Avaliação cefalométrica radiográfica da posição craniocervical de pacientes orientados em posição natural da cabeça pré e pós-expansão rápida da maxila*
}

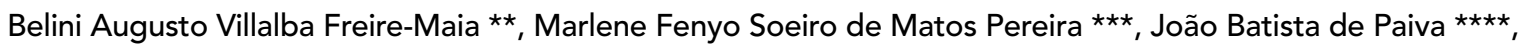

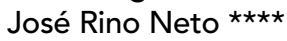

\begin{abstract}
Resumo
O objetivo deste estudo foi avaliar a eventual alteração na posição craniocervical de pacientes orientados na Posição Natural da Cabeça (PNC), após o procedimento de expansão rápida da maxila (ERM). Utilizaram-se telerradiografias em norma lateral, selecionadas do acervo de documentação da Clínica de Ortodontia Preventiva da Faculdade de Odontologia da Universidade de São Paulo, de 20 pacientes, com idades entre 5 a 11 anos (média de 9,0 anos), sendo 7 do gênero masculino e 13 do feminino, obtidas no início do tratamento e imediatamente após a fase ativa da expansão da maxila. Os pacientes eram portadores de atresia da maxila e foram tratados com aparelho disjuntor tipo Biederman modificado. A partir dos cefalogramas pré e pós-ERM foram obtidas as grandezas angulares craniovertical (SN.VER), craniocervical (OPT.SN e CVT.SN), cervicohorizontal (OPT.HOR, CVT.HOR) e o ângulo da curvatura cervical (OPT.CVT). Após análise estatística e interpretação dos resultados obtidos concluímos que a posição da cabeça não apresentou alteração estatisticamente significativa. Não foram observadas diferenças estatisticamente significativas entre os gêneros quando comparadas as fases pré e pós-ERM para as grandezas estudadas. O método de obtenção de telerradiografias laterais em Orientação Natural da Cabeça mostrou-se confiável e reproduzível na amostra estudada, mesmo na presença de intervenção ortodôntica-ortopédica entre as séries de radiografias.
\end{abstract}

Palavras-chave: Ortodontia. Posição natural da cabeça. Cefalometria radiográfica. Expansão rápida da maxila.

* Resumo da dissertação de Mestrado em Ortodontia apresentada à Faculdade de Odontologia da Universidade de São Paulo (FOUSP), 2003.

** Mestre em Ortodontia pela Faculdade de Odontologia - USP; Professor do Curso de Atualização em Ortodontia da FUNDECTO - USP.

*** Professora Livre Docente da Disciplina de Radiologia do Departamento de Estomatologia da Faculdade de Odontologia da USP.

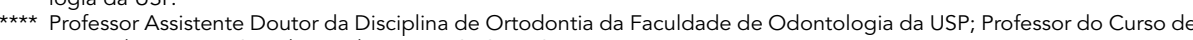
Especialização em Ortodontia da FUNDECTO - USP. 


\section{INTRODUÇÃO}

Os craniologistas, já no século XIX, recomendaram que, para o correto estudo, os crânios deveriam ser orientados de maneira a se aproximar da Posição Natural da Cabeça (PNC) dos vivos. Desde então, vários estudos foram feitos para avaliar a sua reprodutibilidade e compará-la com as linhas de referência intracranianas mais usadas, como o plano horizontal de Frankfurt e a linha Sela-Násio. Uma vez comprovada sua reprodutibilidade, é preferível o uso de uma linha Vertical Verdadeira, ou uma linha perpendicular a ela, às linhas de referência no crânio, já que as variações biológicas das linhas intracranianas estudadas são maiores do que a variação encontrada no registro da PNC 5,8,9,11,13,14,32,38,41,42,45,49.

A utilização de referências intracranianas para a obtenção de telerradiografias e nas análises cefalométricas convencionais pode comprometer o diagnóstico ortodôntico-cirúrgico pelo fato de as estruturas relacionadas apresentarem variações anatômicas significativas ${ }^{5}$. Muitas vezes, o ortodontista se depara com informações conflitantes entre os dados clínicos e cefalométricos. Isso ocorre porque as linhas de referências utilizadas traduzem, não somente as alterações das bases ósseas, mas também expressam variações biológicas que ocorrem na base do crânio.

A PNC se mostrou mais reproduzível quando o paciente afirmava que já estava na posição natural e um profissional treinado corrigia qualquer alteração de cabeça (flexionada ou fletida) que porventura persistisse. A. Lundström e F. Lundström ${ }^{27}$ introduziram, a partir dessa comprovação, o termo Orientação Natural da Cabeça; pois "o clínico e não o paciente é quem determina a posição final".

O controle postural da cabeça é sensível a outros estímulos além daqueles relacionados diretamente à resistência à força da gravidade. Quando a respiração nasal é restabelecida após tonsilectomias, ocorrem alterações favoráveis na postura e mudanças nas dimensões craniofaciais ${ }^{64}$ e em si- mulações de obstrução nasal total com o uso de um tampão, é observada uma extensão na posição da cabeça ${ }^{60}$. Estudos demonstraram alterações na posição espacial da mandíbula pelo uso da placa miorrelaxante superior com conseqüente aumento da dimensão vertical o que foi capaz de alterar a posição da cabeça promovendo sua extensão. A análise cefalométrica de crianças com mordida cruzada que utilizaram aparelho ortodôntico removível também demonstrou aumento na dimensão vertical de oclusão com alteração significativa, mais para anterior, na posição da coluna cervical em relação à Horizontal Verdadeira.

Arcos superiores atrésicos são encontrados com freqüência em indivíduos respiradores bucais. Alguns autores sugerem que a expansão rápida da maxila, além de restabelecer a forma do palato, promove aumento nas dimensões transversais da cavidade nasal e, secundariamente, melhora a função respiratória $4,17,18,15,29,54,55$. Como consequência da expansão, além do restabelecimento da forma do arco superior, ocorre um deslocamento anterior e inferior da maxila, influenciando simultaneamente o posicionamento espacial da mandíbula em ralação à base do crânio, a qual rotaciona para baixo e para trás ${ }^{4}$.

Cientes de que o procedimento da expansão rápida da maxila promove alterações esqueletais importantes na cavidade nasal e no complexo maxilomandibular e da importância da PNC no diagnóstico e planejamento ortodôntico, achamos pertinente avaliar a reprodutibilidade do método de obtenção de telerradiografias em Posição Natural da Cabeça e eventuais alterações no posicionamento craniocervical nas fases pré e pós expansão rápida da maxila.

\section{MATERIAIS E MÉTODOS}

A amostra foi composta de 40 telerradiografias em norma lateral, obtidas de 20 pacientes (aprovado pelo Comitê de Ética em Pesquisa da FOUSP, parecer no 161/02), sendo 7 do gênero masculino e 13 do feminino com idades variando entre 5 anos e 1 mês e 
11 anos e 1 mês (média de 9 anos de idade), tratados na Clínica de Ortodontia Preventiva da Faculdade de Odontologia da Universidade de São Paulo.

Todos os pacientes eram portadores de atresia maxilar, sem agenesias ou mutilações dentárias e foram tratados com aparelho de expansão rápida da maxila tipo Biederman ${ }^{3}$ modificado (Fig. 1, 2), com bandas ortodônticas nos segundos molares decíduos ou primeiros molares permanentes e apoios por lingual nos caninos e primeiros molares decíduos e extensão até o primeiro molar permanente. As ativações do aparelho expansor foram iniciadas uma semana após a instalação do mesmo, com uma volta completa inicial realizada pelo ortodontista. A partir daí, a ativação foi de $1 / 4$ de volta no período da manhã e $1 / 4$ de volta de ativação no período da noite. $\mathrm{O}$ tempo de ativação variou entre 15 e 18 dias, até que se atingisse a expansão desejada, pela abertura da sutura palatina (Fig. 3, 4). A estabilização do parafuso foi feita utilizando-se resina acrílica.

Para a obtenção das telerradiografias em norma lateral foi utilizado aparelho de raios $\mathrm{X}$ modelo Panoura - 10 CSU, marca Yoshida Kaylor, com cefalostato acoplado, com regulagem entre 70 a $80 \mathrm{kVp}, 7 \mathrm{~mA}$ e tempo de exposição de 1,2 segundo. Filme radiográfico Kodak TMG/RA, com dimensões de 18 x $24 \mathrm{~cm}$, com ecrans intensificadores, marca Kodak base verde Lanex. Chassis porta-filme, $18 \times 24 \mathrm{~cm}$. Processadora automática modelo A/T2000 Plus, marca Air Techniques e avental de chumbo.

As telerradiografias em norma lateral de cada paciente foram obtidas pelo mesmo operador, conforme o protocolo da Clínica de Ortodontia Preventiva da Faculdade de Odontologia da Universidade de São Paulo. A primeira foi feita no início do tratamento (Fig. 5), e a segunda, após o término da expansão (Fig. 6), com intervalo de 6 a 10 meses entre elas. Todos os pacientes foram orientados a assumir uma posição natural da cabeça, tendo como referência um espelho posicionado à frente 5 ,8,27,32,38,42,45.
O protocolo utilizado para a obtenção da PNC foi o seguinte:

- paciente em posição ereta, com os pés afastados aproximadamente $10 \mathrm{~cm}$, inclina a cabeça para frente e para trás, diminuindo a amplitude, até sentir que foi alcançado o equilíbrio natural da cabeça (Fig. 7);

- pede-se ao paciente para olhar na imagem refletida de seus olhos, utilizando um espelho oval colocado a sua frente (Fig. 8);

- a posição é então verificada por um profissional experiente, que corrigirá qualquer desvio que possa ter ocorrido na posição da cabeça;

- as olivas auriculares são inseridas no trágus cartilaginosus, mantendo leve contato com a pele, para evitar que o paciente eleve sua cabeça e pescoço;

- posicionador násion é adaptado suavemente, para estabilização no plano vertical. Desta forma, são obtidos três pontos de contato, mantendo a PNC (Fig. 9);

- a linha Vertical Verdadeira é obtida utilizando-se uma corrente metálica unida a um prumo, posicionados próximo à margem anterior do chassi porta-filme, de maneira que apareça à frente do contorno do perfil dos tecidos moles (Fig. 10);

- após a checagem completa dos itens anteriores, obtém-se a telerradiografia em norma lateral.

\section{Obtenção do cefalograma}

Os traçados dos cefalogramas foram realizados com lapiseira Pentel P203, com grafite preto e diâmetro de $0,3 \mathrm{~mm}$, em papel Ultraphan, de 0,07 mm de espessura e dimensões de 17,5 x 17,5 $\mathrm{cm}$, fixados com fita adesiva sobre a telerradiografia em norma lateral. Para melhor visualização das estruturas anatômicas foram feitos em uma sala totalmente escura com o auxílio de um negatoscópio de mesa.

As estruturas anatômicas craniofaciais foram traçadas como preconizado por Interlandi ${ }^{22}$ e Vigorito $^{61}$, e as cervicais segundo Solow e Tallgren ${ }^{49}$ (Fig. 11), são elas: perfil dos tecidos moles; sela turca; perfil anterior do osso frontal e dos ossos nasais; 


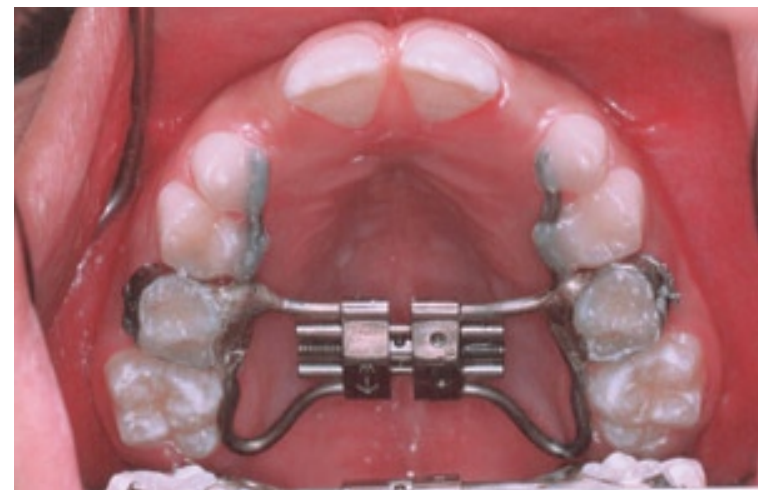

FIGURA 1 - Aparelho disjuntor da maxila tipo Biederman modificado, pré-ERM.

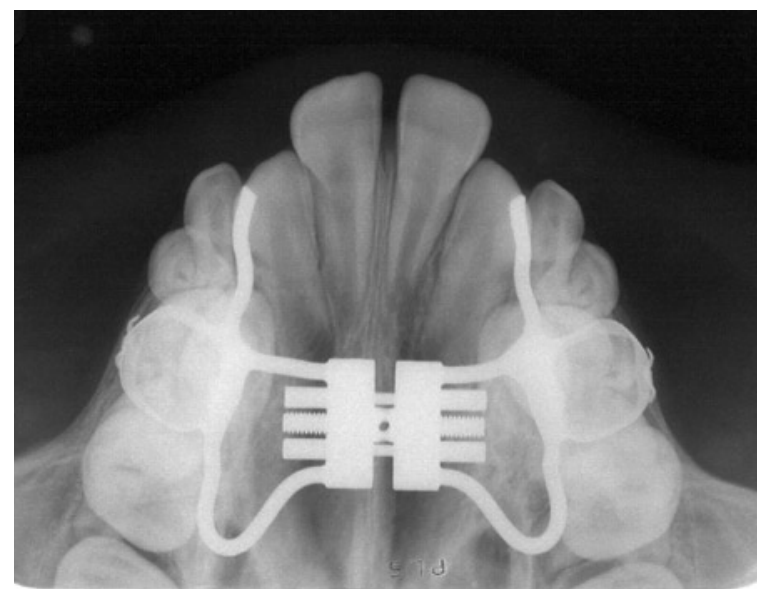

FIGURA 3 - Radiografia oclusal da maxila, pré-ERM.

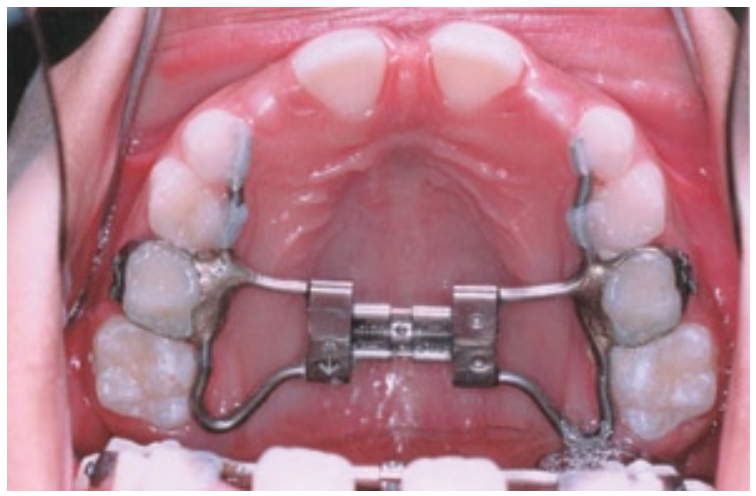

FIGURA 2 - Aparelho disjuntor da maxila tipo Biederman modificado, pós-ERM.

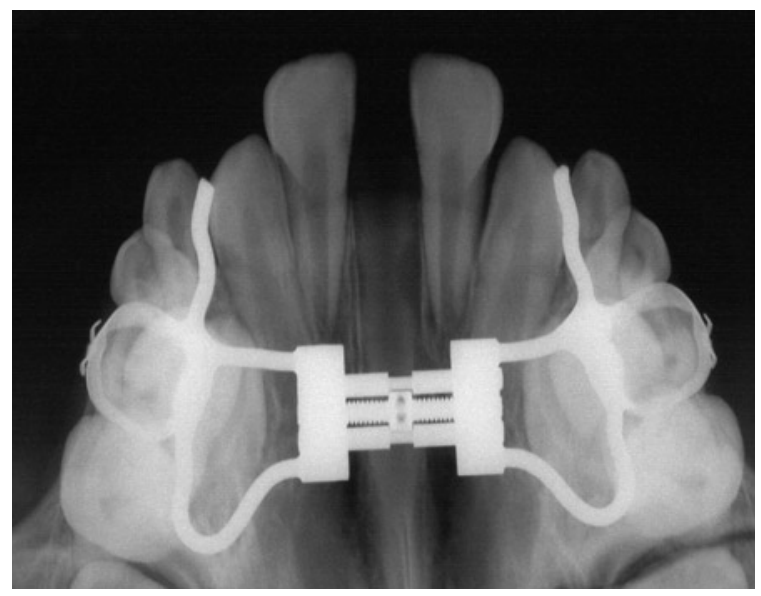

FIGURA 4 - Radiografia oclusal da maxila, pós-ERM.

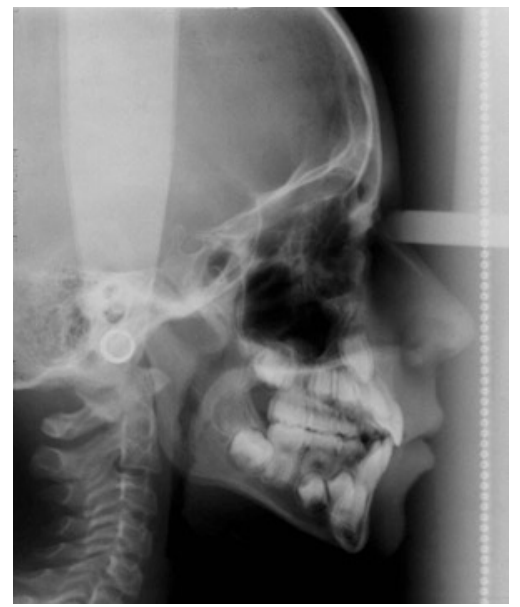

FIGURA 5 - Telerradiografia em norma lateral, pré-ERM.

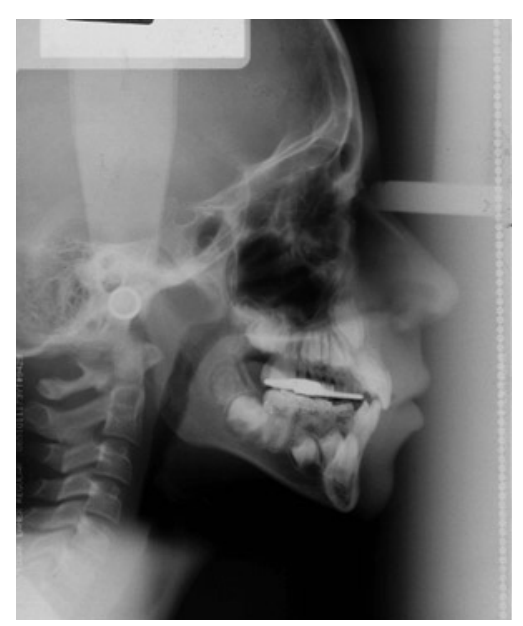

FIGURA 6 - Telerradiografia em norma lateral, pós-ERM.

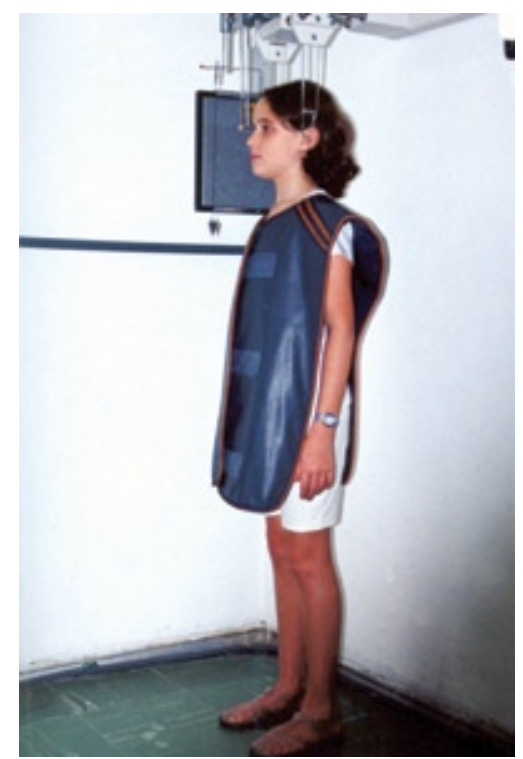

FIGURA 7 - Posicionamento da paciente em pé, com os pés ligeiramente afastados. 


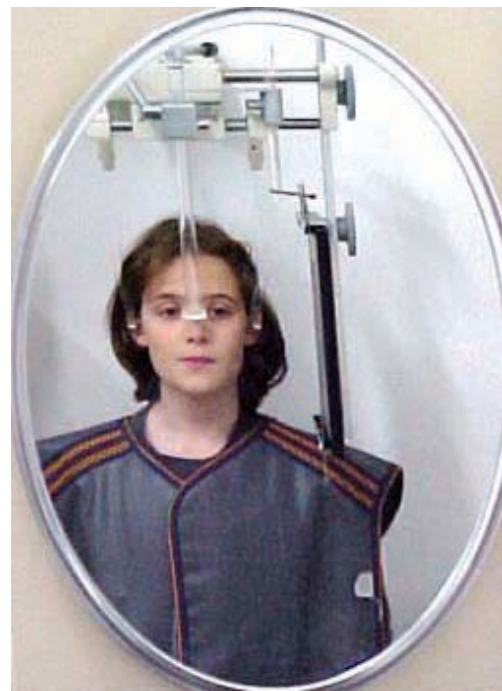

FIGURA 8 - Imagem refletida da paciente visualizando os próprios olhos em um espelho oval.

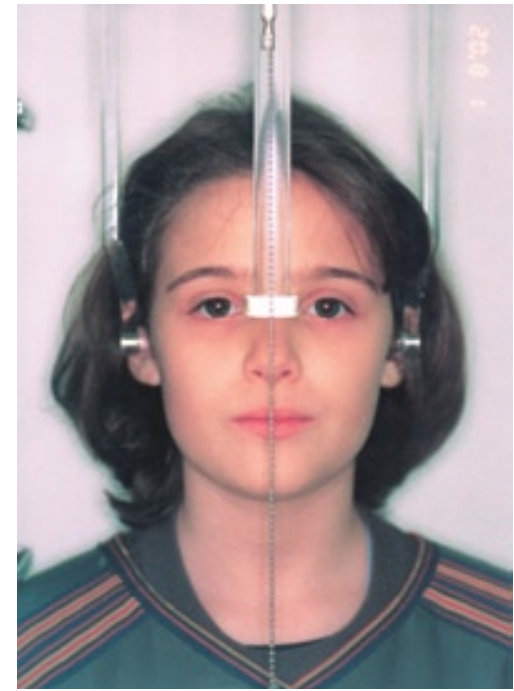

FIGURA 9 - Posicionador násion e olivas auriculares adaptados. A linha bipupilar deve estar paralela ao solo.

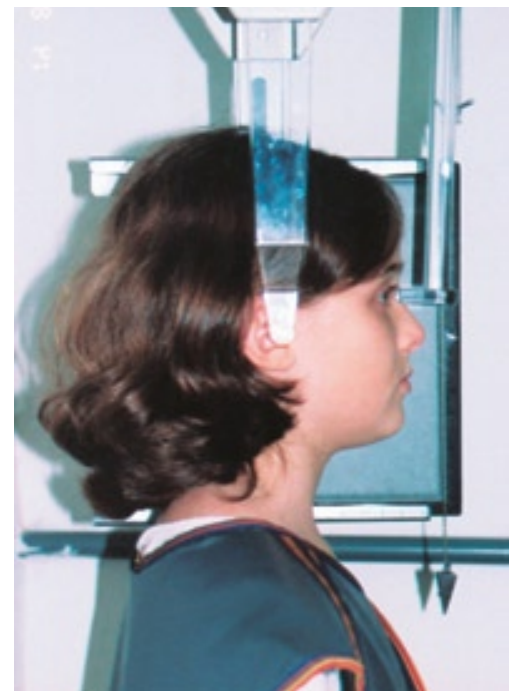

FIGURA 10 - Corrente metálica posicionada próximo ao filme (VER). As pupilas devem se encontrar no centro do globo ocular.

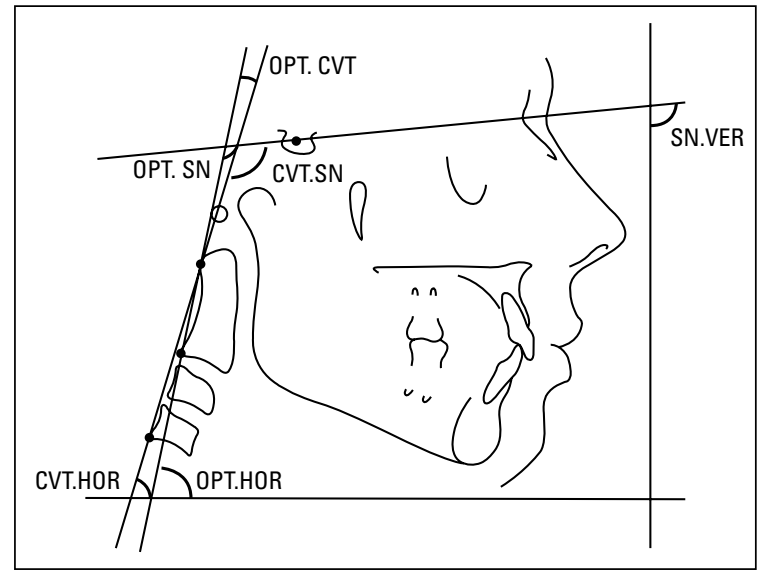

FIGURA 11 - Desenho anatômico, pontos cefalométricos, linhas cefalométricas, grandezas cefalométricas.

meato acústico externo; borda póstero-inferior das órbitas; maxila; mandíbula; incisivos centrais superiores e inferiores; $1{ }^{\text {os }}$ molares permanentes, superiores e inferiores; processo odontóide da segunda vértebra cervical e corpos da terceira e quarta vértebras cervicais.

\section{Pontos cefalométricos}

Os seguintes pontos cefalométricos foram traçados (Fig. 11):

- Sela (S): localizado no centro da sela túrcica.
- Násio $(\mathrm{N})$ : ponto mais anterior da sutura fronto-nasal.

- cv 2tg: ponto por onde passa a linha tangente ao processo odontóide, na região póstero-superior da segunda vértebra cervical.

- cv 2ip: ponto mais ínfero-posterior do corpo da segunda vértebra cervical.

- cv 4ip: ponto mais ínfero-posterior do corpo da quarta vértebra cervical.

\section{Linhas cefalométricas}

Foram traçadas as seguintes linhas cefalométricas (Fig. 11).

- SN: união dos pontos sela-násio.

- OPT: tangente ao processo odontóide. Linha tangente posterior ao processo odontóide que passa pelo ponto cr $2 \mathrm{ip}$.

- CVT: tangente à vértebra cervical. Linha tangente posterior ao processo odontóide passando pelo ponto cv $4 \mathrm{ip}$.

- VER: linha Vertical Verdadeira. A linha vertical traçada a partir da imagem do prumo projetada no filme radiográfico.

- HOR: linha Horizontal Verdadeira perpendicular à VER. 


\section{Grandezas cefalométricas}

As grandezas cefalométricas que foram utilizadas são as seguintes (Fig. 11):

- SN.VER: ângulo anterior e inferior formado pela intersecção das linhas sela-násio e vertical verdadeira. Inclinação da cabeça em relação à VER.

- OPT.SN: ângulo formado pela intersecção da linha tangente ao processo odontóide e a linha sela-násio. Inclinação da cabeça em relação à coluna cervical.

- CVT.SN: ângulo formado pela intersecção da linha tangente à vértebra cervical e a linha selanásio. Inclinação da cabeça em relação à coluna cervical.

- OPT.CVT: ângulo formado pela intersecção da linha tangente ao processo odontóide e a linha tangente à vértebra cervical. Representa o grau de curvatura cervical.

- OPT.HOR: ângulo formado pela intersecção da linha tangente ao processo odontóide e à linha horizontal verdadeira. Permite avaliar a inclinação da coluna cervical em relação à linha horizontal verdadeira.

- CVT.HOR: ângulo formado pela intersecção da linha tangente à vértebra cervical com a horizontal verdadeira. Avalia a inclinação da coluna cervical em relação à linha horizontal verdadeira.

\section{Erro do método}

Para se verificar o erro do método, os cefalogramas e todas as medidas obtidas das telerradiografias em norma lateral da amostra (pré e pós-ERM) foram repetidos pelo mesmo operador, aproximadamente dois meses após a primeira mensuração. Verificou-se, desta forma, a concordância entre as medidas pela correlação intra-classe ${ }^{12}$.

\section{Método estatístico}

Foram calculados os valores das médias e dos erros padrão. Para se verificar se houve alterações no posicionamento da cabeça após o uso do aparelho Biederman modificado, foi utilizada a técnica de análise de variância com medidas repetidas $\mathrm{ANOVA}^{48}$, e foi utilizado mais um fator fixo, o gênero, para cada medida angular realizada. Foram realizadas comparações múltiplas de Bonferroni ${ }^{35}$ quando o comportamento dos momentos das medidas fosse diferente para cada gênero, a fim de se verificar onde ocorreram essas diferenças. O nível de significância adotado foi de 0,05 .

Aplicou-se a fórmula de Dahlberg ${ }^{19}$ para avaliar o erro do método (reprodutibilidade) da posição da cabeça entre os diferentes períodos de obtenção das telerradiografias, para SN.VER:

$$
\sqrt{\Sigma\left(d^{2} / 2 n\right)}
$$

\section{RESULTADOS}

Estão expostos na tabela 1 as médias e os erros padrão para cada variável estudada, pré e pós ERM, considerando-se o gênero.

Os resultados da análise de variância com medidas repetidas (ANOVA) nas fases pré e pós-ERM e se houve influência do gênero nas variáveis SN.VER, OPT.SN, CVT.SN e OPT.CVT estão na tabela 2. Na tabela 3 estão os resultados da análise de variância (ANOVA) para os ângulos OPT.HOR e CVT.HOR. Como houve mudanças de comportamento pré e pós-ERM nos dois gêneros, foram feitas comparações múltiplas de Bonferroni para determinar onde ocorreram tais alterações.

O erro do método (reprodutibilidade) encontrado, obtido empregando-se a fórmula de Dahlberg para avaliar a variação da posição da cabeça entre os diferentes períodos de obtenção das telerradiografias, para a variável SN.VER, foi de $2,9149^{\circ}$.

\section{DISCUSSÃO}

A Cefalometria radiográfica, que se desenvolveu a partir da introdução do cefalostato por Broadbent ${ }^{6}$ em 1931, é notoriamente um excelente elemento de diagnóstico, mas dependendo das referências intracranianas utilizadas pode induzir o ortodontista a interpretações equivocadas.

A variação dessas referências foi claramente 


\begin{tabular}{|c|c|c|c|c|}
\hline Ângulo & ERM & Gênero & Média & Erro Padrão \\
\hline \multirow{4}{*}{ SN.VER } & Pré & Masculino & 98.86 & 2.1287 \\
\hline & Pós & Masculino & 99.25 & 1.4255 \\
\hline & Pré & Feminino & 97.98 & 1.5621 \\
\hline & Pós & Feminino & 99.04 & 1.0460 \\
\hline \multirow{4}{*}{ OPT.SN } & Pré & Masculino & 94.50 & 3.3141 \\
\hline & Pós & Masculino & 98.89 & 2.9167 \\
\hline & Pré & Feminino & 97.52 & 2.4319 \\
\hline & Pós & Feminino & 95.29 & 2.1402 \\
\hline \multirow{4}{*}{ CVT.SN } & Pré & Masculino & 96.70 & 2.9287 \\
\hline & Pós & Masculino & 100.83 & 2.7282 \\
\hline & Pré & Feminino & 102.40 & 2.1491 \\
\hline & Pós & Feminino & 100.38 & 2.0019 \\
\hline \multirow{4}{*}{ OPT.CVT } & Pré & Masculino & 3.43 & 0.9245 \\
\hline & Pós & Masculino & 3.32 & 0.9655 \\
\hline & Pré & Feminino & 4.90 & 0.6784 \\
\hline & Pós & Feminino & 5.08 & 0.7085 \\
\hline \multirow{4}{*}{ OPT.HOR } & Pré & Masculino & 94.04 & 2.4443 \\
\hline & Pós & Masculino & 89.39 & 2.0685 \\
\hline & Pré & Feminino & 90.33 & 1.7937 \\
\hline & Pós & Feminino & 92.92 & 1.5179 \\
\hline \multirow{4}{*}{ CVT.HOR } & Pré & Masculino & 91.82 & 2.0172 \\
\hline & Pós & Masculino & 87.39 & 1.8953 \\
\hline & Pré & Feminino & 85.40 & 1.4802 \\
\hline & Pós & Feminino & 87.85 & 1.3908 \\
\hline
\end{tabular}

descrita por Moorrees e Kean ${ }^{32}$, quando compararam dois cefalogramas com perfis faciais semelhantes e observaram grande diferença entre a linha SN e PHF. Posteriormente, Moorrees ${ }^{33}$ quantificou essas diferenças, sendo de $12.5^{\circ}$ e $7^{\circ}$, respectivamente. É notório que essas variações estarão embutidas nas grandezas cefalométricas, comprometendo assim o diagnóstico e, conseqüentemente, o plano de tratamento ${ }^{5,10,20,27,32,33,36,59}$. Isto, porém, pode ser evitado utilizando-se a Posição Natural da Cabeça e relacionando-se as medidas às linhas Horizontal e Vertical Verdadeiras, ${ }^{70,36}$.

Downs $^{10}$, Bjerin ${ }^{5}$, Moorrees e Kean ${ }^{32}$, baseados

\begin{tabular}{|c|c|c|c|c|}
\hline \multicolumn{5}{|c|}{ Tabela 2 - Análise de variância (p) para os ângulos: } \\
& SN.VER, OPT.SN, CVT.SN e OPT.CVT. \\
\hline & SN.VER & OPT.SN & CVT.SN & OPT.CVT \\
\hline $\begin{array}{c}\text { Gênero } \\
\text { Fase da } \\
\text { ERM }\end{array}$ & 0.7927 & 0.9348 & 0.4193 & 0.1537 \\
\hline $\begin{array}{c}\text { Gênero/fase } \\
\text { da ERM }\end{array}$ & 0.4522 & 0.5076 & 0.4867 & 0.9418 \\
\hline & 0.7288 & 0.0530 & 0.0536 & 0.7566 \\
\hline
\end{tabular}

\begin{tabular}{|c|c|c|c|c|c|}
\hline \multicolumn{6}{|c|}{ Gênero/fase da ERM } \\
\hline \multicolumn{3}{|c|}{ Ângulo } & \multicolumn{3}{|c|}{$\mathbf{p}$} \\
\hline \multicolumn{3}{|c|}{ OPT.HOR } & \multicolumn{3}{|c|}{0.0167} \\
\hline \multicolumn{3}{|c|}{ CVT.HOR } & \multicolumn{3}{|c|}{0.0125} \\
\hline $\begin{array}{c}\text { Fase da } \\
\text { ERM }\end{array}$ & Gênero & $\begin{array}{c}\text { Fase da } \\
\text { ERM }\end{array}$ & Gênero & $\begin{array}{c}\mathbf{p} \\
\text { (OPT. } \\
\text { HOR) }\end{array}$ & $\begin{array}{c}\mathbf{p} \\
\text { (CVT. } \\
\text { HOR) }\end{array}$ \\
\hline Pré & Masc. & Pós & Masc. & 0.3019 & 0.2379 \\
\hline Pré & Masc. & Pré & Fem. & 1.0000 & 0.1169 \\
\hline Pré & Masc. & Pós & Fem. & 1.0000 & 0.7325 \\
\hline Pós & Masc. & Pré & Fem. & 1.0000 & 1.0000 \\
\hline Pós & Masc. & Pós & Fem. & 1.0000 & 1.0000 \\
\hline Pré & Fem. & Pós & Fem. & 0.7640 & 0.6770 \\
\hline
\end{tabular}

em estudos craniométricos, propuseram o emprego de referências extracranianas obtidas a partir de indivíduos em PNC, tendo como justificativa a necessidade na determinação de um plano de referência craniofacial para valorizar a estética facial em análises ortodônticas e no planejamento dos tratamentos. Entretanto, o conceito não é novo, já que foi descrito pela primeira vez por Broca (1862, apud COOKE, $\mathrm{WEI}^{8}, 1988$ ), como sendo a posição adotada por um homem em pé com seu eixo visual situado no plano horizontal. Esta é a posição em que o indivíduo assume no dia a dia e não o plano horizontal de Frankfurt paralelo ao solo 59 . 
Há, descritos na literatura, diferentes métodos preconizados para o registro da Posição Natural da Cabeça. Dentre os mais citados, encontramos o método do espelho, no qual os indivíduos são solicitados a olhar para a imagem de seus olhos refletida em um espelho localizado no nível das pupilas $^{5,16,25,32,40,42,51,52}$; o método do auto-equilíbrio ${ }^{49}$, obtido pela própria sensação de equilíbrio da cabeça do indivíduo, sem a utilização de qualquer referência externa; e há ainda uma combinação de ambos $^{42,45}$. Rink et al. ${ }^{40}$ e Viazis ${ }^{59}$, no entanto, não utilizaram qualquer objeto em especial, pedindo apenas para o paciente olhar para a linha do horizonte. Esse método também foi utilizado por Showfety et al. ${ }^{44}$, que solicitavam aos indivíduos que olhassem um ponto distante como se avistassem uma longa estrada no horizonte através da janela.

Com relação às diferenças entre os gêneros no registro da $\mathrm{PNC}$, Cooke e $\mathrm{Wei}^{8}$, no estudo com indivíduos chineses, observaram apenas em um dos oito grupos avaliados que os meninos olharam mais para cima quando foi utilizado o espelho como fonte externa de referência para os olhos em relação à posição de auto-equilíbrio, na qual adotam uma postura de cabeça mais relaxada. Nas meninas, não foram observadas alterações, possivelmente porque elas são estimuladas socialmente a adotar uma postura mais ereta, e provavelmente continuam a adotar essa postura mesmo na ausência da referência externa para os olhos. Nenhuma outra diferença entre os gêneros na reprodutibilidade da PNC foi encontrada, fato que foi confirmado posteriormente no estudo longitudinal por Peng e Cooke $^{38}$, também com indivíduos chineses.

Quanto à posição dos indivíduos (sentados ou em pé) para o registro da PNC, Moorrees e $\mathrm{Kean}^{32}$ preferiam que eles ficassem sentados de maneira confortável numa banqueta, enquanto Viazis $^{59}$ mencionou que os pacientes devem ficar sentados, em posição ereta, para a obtenção da telerradiografia em norma lateral. Porém, de acordo com a literatura, a posição mais utilizada é a em pé $^{24,26,27,38,49,50,51}$. Como visto, apesar de Bjerin ${ }^{5}$, já em 1957, ter encontrado um desvio padrão menor para a posição em pé $\left(1,34^{\circ}\right)$ do que para a posição sentada $\left(1,62^{\circ}\right)$, não há consenso na literatura a respeito de qual a melhor posição do indivíduo no cefalostato.

A possibilidade da utilização de linhas de referência extracranianas, como a Horizontal e a Vertical Verdadeira, para estudos cefalométricos, só é possivel porque foi confirmada a hipótese de que a PNC é relativamente constante no ser humano (reprodutibilidade) ${ }^{5,8,11,13,14,32,41,42,45,49}$ e permanece válida ao longo do tempo ${ }^{9,38}$.

A variação encontrada para as linhas intracranianas (D.P. de $3,55^{\circ}$ a $6,69^{\circ}$ ) é maior do que a variação (D.P. $=1,54^{\circ}$ ) para o registro da posição da cabeça ${ }^{32}$. A obtenção de radiografias cefalométricas em Posição Natural da Cabeça e o uso de uma linha Vertical Verdadeira como referência são mais confiáveis do que o uso rotineiro de algumas linhas, como o plano Horizontal de Frankfurt ou a linha Sela-Násio. As variações biológicas das linhas intracranianas foram maiores do que a variação encontrada no registro da PNC, fato corroborado por Cooke e $\mathrm{Wei}^{7}$, que relataram variação das linhas de referências intracranianas em relação à Vertical Verdadeira em PNC de $25^{\circ}$ a $36^{\circ}$ $\left( \pm 5^{\circ}\right.$ a $\left.6^{\circ}\right)$, enquanto que a reprodutibilidade em PNC foi de $4^{\circ}$. Os autores recomendaram a Posição Natural da Cabeça para registros cefalométricos e o uso da linha Vertical Verdadeira como linha de referência básica, na análise da morfologia facial e na avaliação do desenvolvimento facial. Portanto, as pequenas diferenças no registro da Posição Natural da Cabeça constituem atualmente um pequeno problema, comparado à variação das linhas de referências intracranianas. Se o paciente for posicionado com critério, de maneira que a cabeça esteja na posição natural, o uso das linhas Vertical ou Horizontal Verdadeiras tem mais credibilidade do que se forem utilizadas as linhas de referências intracranianas ${ }^{13,26}$.

O posicionamento do paciente no cefalostato deve ser criterioso, pois alterações na postura da 
cabeça podem produzir efeitos significativos em algumas grandezas cefalométricas, já que afetam a localização de alguns pontos cefalométricos e os dados subseqüentes ${ }^{30,56}$. Além disso, de acordo com Moorrees e Kean ${ }^{32}$, o método de posicionamento dos pacientes em PNC demonstrou menor variação quando a posição da cabeça era estimada por um clínico treinado. Desta maneira, a Orientação Natural da Cabeça (ONC), proposta por Lundström et al. ${ }^{26}$, deve ser aplicada para se evitar qualquer tendência do paciente em manter a cabeça numa posição não natural (flexionada ou estendida), sendo o clínico, e não o paciente, quem vai determinar a posição final.

A posição da cabeça demonstrou ser influenciada por fatores funcionais, tais como a ausência de visão ${ }^{11}$ e a respiração bucal, que mostrou promover sua extensão $0^{21,43,60}$.

Ricketts $^{39}$ descreveu o quadro de um indivíduo com síndrome da obstrução respiratória, o qual estendia a cabeça para promover o aumento do espaço aéreo e, conseqüentemente, aumentar o fluxo de ar, fato confirmado posteriormente por Solow e Tallgren ${ }^{50}$. Essa extensão deve ser significativa, pois, de acordo com Weber et al. ${ }^{63}$, até 10 graus de extensão da cabeça, a partir da postura de repouso normal, não há alteração significativa da resistência do espaço aéreo superior, durante a inspiração ou a expiração. Para Ricketts ${ }^{39}$, uma vez realizada a adenoidectomia, eliminando-se dessa forma a obstrução do espaço aéreo da nasofaringe, a respiração passava de bucal para nasal e ocorriam, conseqüentemente, mudanças na postura da cabeça. Mesmo Tourne e Schweiger ${ }^{57}$, que não observaram extensão da cabeça significativa após um experimento de obstrução respiratória nasal através da utilização de um grampo no nariz por uma hora, acreditaram que as respostas posturais poderiam ser diferentes nos casos em que houvesse presença excessiva de tecido linfóide.

A ortoposição, definida por Molhave (1958, apud SOLOW, TALLGREN ${ }^{49}$, 1971) como a posição intencional da vertical ereta para a móvel (indivíduo anda suavemente no lugar), também se mostrou influenciada pela função respiratória. Apesar de reproduzivel para a mesma pessoa, pode diferir entre indivíduos, bastando apenas que exista obstrução das vias aéreas superiores ${ }^{34}$.

As funções orais, entre elas a respiração bucal, constituem fatores etiológicos em potencial na deterioração da oclusão e na alteração do padrão normal de crescimento facial ${ }^{46}$, pois requerem numerosas adaptações musculares, entre as quais elevação da cabeça, com hiperextensão anterior do pescoço; e abaixamento da mandíbula, acompanhada da língua que, nessa posição, não se contrapõe à força sagital da musculatura peribucal, podendo propiciar a atresia da maxila ${ }^{28,39,53}$.

Os aparelhos de expansão rápida da maxila, propostos por Haas ${ }^{15}$ e Biederman ${ }^{3}$, possuem efeitos similares e eficazes para promover a correção da mordida cruzada e da atresia maxilar por meio da abertura da sutura palatina mediana ${ }^{23}$. Esse fato levantou a hipótese de que o aumento da dimensão transversal da maxila levaria conseqüentemente ao aumento do espaço da cavidade nasal, comprovado pela redução na resistência aérea da ordem de $36,2 \%$, tornando possivel que indivíduos respiradores bucais passassem a realizar a respiração nasal $1^{4,17,18,15,29,54,55}$.

Wertz ${ }^{62}$ observou que a abertura da sutura palatina mediana, com o único propósito de aumentar a permeabilidade nasal, não pode ser justificada, a menos que a obstrução esteja localizada na região ântero-inferior da cavidade e acompanhada pela atresia do arco dentário superior. Já Paiva et al. ${ }^{37}$ não observaram aumento significativo na fase pósexpansão rápida, comparado à fase pré-expansão rápida da maxila, mas salientaram que pacientes com atresia da maxila não apresentam necessariamente algum fator obstrutivo na cavidade nasal, e conseqüentemente, nesses casos, a respiração não se beneficiaria diretamente da ERM.

Há estreita relação entre mudanças na postura da cabeça e no posicionamento da mandíbula - o aumento da dimensão vertical está 
associado com extensão da cabeça ${ }^{2,58}$. A ERM tem, como efeito secundário, o deslocamento inferior da maxila e, conseqüentemente, a rotação da mandíbula em sentido horário ${ }^{1,47}$. Dessa forma, seria de se esperar que a utilização de aparelhos que promovam a abertura da sutura palatina mediana induziriam mudanças espaciais na posição da cabeça, no caso a sua extensão; apesar de Solow e Sonnesen ${ }^{52}$ não terem observado associação entre discrepâncias transversais e os ângulos posturais. Mesmo os aparelhos expansores removíveis, cujo efeito é de causar expansão lenta da maxila, também demonstraram aumentar a dimensão vertical de oclusão, promovendo, dessa forma, alteração significativa na posição da coluna cervical (mais para anterior). Em contrapartida, não foi encontrada alteração significativa na extensão da cabeça em relação à linha Vertical Verdadeira ${ }^{31}$.

Partindo do princípio de que a Posição Natural da Cabeça é altamente reprodutível em crianças brasileiras, como comprovado por Rino Neto et al. ${ }^{41}$, e que a posição da cabeça pode ser afetada pelos diversos fatores anteriormente discutidos, tivemos a intenção, no presente estudo, de avaliar se a posição da cabeça (sob orientação), mesmo na presença de algum tipo de intervenção ortodôntica-ortopédica - no caso a ERM - mostraria alguma alteração significativa que pudesse comprometer a utilização da PNC como método de diagnóstico, e sua eventual relação com o fator gênero.

Discutiremos, a seguir, as variáveis SN.VER, OPT.SN, CVT.SN, OPT.CVT, OPT.HOR, CVT. HOR e a reprodutibilidade do método de registro da PNC utilizado neste estudo.

\section{Ângulo craniovertical (SN.VER)}

Ângulo que representa a posição da cabeça em relação à linha vertical verdadeira (VER). Ocorre extensão da cabeça (inclinação para cima) quando há aumento deste ângulo, e flexão (inclinação para baixo) quando há diminuição. Solow e Tallgren ${ }^{49}$ encontraram, para esta grandeza, o valor médio de $92,59^{\circ}( \pm 4,73)$ para os indivíduos posicionados em PNC pelo método do espelho.

Os valores encontrados neste estudo para as médias e erros padrão pré e pós-ERM (Tab. 1) foram os seguintes:

- Gênero masculino: $98,86^{\circ}( \pm 2,13)$ e $99,25^{\circ}$ $( \pm 1,43)$, respectivamente.

- Gênero feminino: $97,98^{\circ}( \pm 1,56)$ e $99,04^{\circ}$ $( \pm 1,05)$, respectivamente.

Pela análise de variância com medidas repetidas (ANOVA) (Tab. 2) o gênero não influenciou nas alterações dos valores dos ângulos pré e pós-ERM e as alterações encontradas na posição da cabeça com a utilização do aparelho não foram estatisticamente significativas, apesar de 13 crianças terem estendido e 4 flexionado suas cabeças na $2^{\text {a }}$ série de radiografias quando comparada aos dados da $1^{\text {a }}$ série.

Os resultados deste estudo são similares aos de Miralles et $a .^{31}$, que não encontraram alterações significativas na posição da cabeça em relação à VER em indivíduos que usaram aparelho expansor removível após quatro meses (inicial $=92,78^{\circ}$ e final $=94,47^{\circ}$, em média). Por outro lado, Andrighetto et al. ${ }^{2}$, após a desprogramação neuromuscular pelo uso de placa miorrelaxante superior, observaram extensão significativa da cabeça em relação à VER em indivíduos posicionados em Posição Natural da Cabeça, mas não pelo método de ONC.

\section{Ângulos craniocervicais (OPT.SN e CVT.SN)}

Ângulos que representam a inclinação da cabeça em relação à coluna cervical. Aumentos nestes ângulos indicam extensão da cabeça sobre a coluna cervical, e diminuições, flexão. Solow e Tallgren ${ }^{49}$ encontraram os valores médios, para o ângulo OPT.SN, $92,18^{\circ}( \pm 6,00)$ e para o ângulo CVT.SN, $97,72^{\circ}( \pm 5,65)$.

Neste estudo, os valores encontrados das médias e erros padrão para o ângulo OPT.SN pré e pós-ERM (Tab. 1) foram os descritos a seguir. 
- Gênero masculino: $94,50^{\circ} \quad( \pm 3,31)$ e $98,89^{\circ}( \pm 2,92)$, respectivamente.

- Gênero feminino: 97,52 $( \pm 2,43)$ e $95,29^{\circ}( \pm 2,14)$, respectivamente.

As médias e erros padrão pré e pós-ERM para o ângulo CVT.SN (Tab. 1) foram:

- Gênero masculino: $96,70^{\circ}( \pm 2,93)$ e $100,83^{\circ}( \pm 2,73)$, respectivamente.

- Gênero feminino: $102,40^{\circ} \quad( \pm 2,15)$ e $100,38^{\circ}( \pm 2,00)$, respectivamente.

Pelo teste de ANOVA (Tab. 2), o gênero não influenciou nas alterações dos valores dos ângulos pré e pós-ERM para ambas as grandezas (OPT.SN e CVT.SN). Apesar de terem ocorrido alterações na posição da cabeça em relação à coluna cervical, após a utilização do aparelho em todas as crianças, as diferenças não foram estatisticamente significativas.

Para Andrighetto et al. ${ }^{2}$, houve extensão significativa da cabeça em relação à coluna cervical após a desprogramação neuromuscular, enquanto que Miralles et al. ${ }^{31}$ não observaram tal fato após o aumento da DV pelo uso de aparelho removível, resultado similar ao encontrado neste estudo.

\section{Ângulo da curvatura cervical (OPT.CVT)}

Ângulo que representa o grau de alteração na curvatura cervical. Aumentos nestes ângulos indicam maior profundidade na curvatura cervical, e vice versa. Solow e Tallgren ${ }^{49}$ encontraram para esse ângulo valor médio de $5,54^{\circ}( \pm 2,57)$.

Em nosso estudo, os valores encontrados das médias e erros padrão para o ângulo OPT.CVT pré e pós ERM (Tab. 1) foram:

- Gênero masculino: $3,43^{\circ} \quad( \pm 0,92)$ e $3,32^{\circ}( \pm 0,97)$, respectivamente.

- Gênero feminino: $4,90^{\circ} \quad( \pm 0,68)$ e $5,08^{\circ}( \pm 0,71)$, respectivamente.

Pelo teste ANOVA (Tab. 2), o gênero não influenciou nas alterações dos valores dos ângulos pré e pós-ERM. As alterações encontradas na profundidade da curvatura cervical com a utilização do aparelho também não foram estatisticamente significativas, resultado semelhante ao observado por Andrighetto et al. ${ }^{2}$ em pacientes que usaram placa miorrelaxante.

\section{Ângulos cervicohorizontais (OPT.HOR e CVT.HOR)}

São os ângulos que representam a relação entre a coluna cervical e a linha Horizontal Verdadeira. $\mathrm{O}$ aumento de seus valores significa que a coluna cervical está mais inclinada em direção posterior em relação à linha Horizontal Verdadeira e a sua diminuição para a anterior. Solow e Tallgren ${ }^{49}$ encontraram, para OPT.HOR, o valor médio de $90,41^{\circ}( \pm 5,89)$, e a média de $84,87^{\circ}( \pm 5,19)$, para o ângulo CVT.HOR,

Neste estudo, os valores encontrados das médias e erros padrão para o ângulo OPT.HOR pré e pós-ERM (Tab. 1) foram:

- Gênero masculino: $94,04^{\circ}( \pm 2,44)$ e $89,39^{\circ}( \pm 2,07)$, respectivamente.

- Gênero feminino: 90,33 $( \pm 1,79)$ e $92,92^{\circ}( \pm 1,52)$, respectivamente.

As médias e erros padrão pré e pós-ERM para o ângulo CVT.HOR (Tab. 1) foram:

- Gênero masculino: $91,82^{\circ}( \pm 2,02)$ e $87,39^{\circ}( \pm 1,90)$, respectivamente.

- Gênero feminino: $85,40^{\circ} \quad( \pm 1,48)$ e $87,85^{\circ}( \pm 1,39)$, respectivamente.

As alterações encontradas na inclinação da coluna cervical em relação à linha Horizontal Verdadeira não foram estatisticamente significativas. Embora similar ao encontrado por Andrighetto et $\mathrm{al}^{2}$, após a desprogramação neuromuscular, nosso resultado foi contrário ao observado por Miralles et al. ${ }^{31}$, que constataram alteração significativa na posição da coluna cervical, estando numa posição mais para anterior em relação à linha Horizontal Verdadeira, em pacientes que utilizaram aparelho expansor removível.

O teste de análise de variância com medidas repetidas (ANOVA) (Tab. 3) indicou que ocorreram alterações nos valores dos ângulos entre os 
gêneros antes e após o uso do aparelho para os dois ângulos, OPT.HOR ( $\mathrm{p}=0,0167)$ e CVT.HOR $(\mathrm{p}=0,0125)$. Utilizando, porém, as comparações múltiplas de Bonferroni, não foi possível verificar diferenças estatisticamente significativas.

\section{Reprodutibilidade do método}

A importância da reprodutibilidade da PNC advém do fato de possibilitar a utilização de linhas extracranianas para a realização de estudos cefalométricos e checar a confiabilidade das linhas de referência intracranianas. Na literatura, o ângulo mais utilizado é o SN.VER (Fig. 11), formado pela intersecção das linhas Sela-Násio e Vertical Verdadeira, obtida a partir da imagem radiográfica de uma corrente metálica posicionada à frente do contorno do perfil dos tecidos moles. São obtidas duas radiografias em períodos distintos, medidos os ângulos SN.VER e então calculada a reprodutibilidade do método utilizando-se a fórmula de Dahlberg.

O valor encontrado neste estudo para o ângulo SN.VER foi de $2.91^{\circ}$, valor próximo aos encontrados nos estudos de Moorrees e Kean ${ }^{32}$ $\left(1.54^{\circ}\right)$ e de Rino Neto et $\mathrm{ll}^{.41}\left(1.15^{\circ}\right)$, nos quais a Posição Natural da Cabeça também foi orientada. Os valores dos estudos são muito próximos e refletem a confiabilidade do método, visto serem considerados aceitáveis valores de até 4 graus ${ }^{59}$.

\section{CONSIDERAÇÕES FINAIS}

$\mathrm{Na}$ busca da excelência do conhecimento das relações dentofaciais aplicadas ao diagnóstico ortodôntico e cirúrgico, destacamos a importância de a avaliação cefalométrica em norma lateral ser realizada com o paciente em Posição Natural da Cabeça. Dentre os aspectos abordados no presente trabalho, inicialmente pode-se observar que a reprodução da posição da cabeça para a obtenção de telerradiografias em norma lateral pode ser conseguida por técnicos de radiologia, com instruções simples de posicionamento ao paciente. Porém, a reprodutibilidade da PNC é maior quando essa posição é orientada por um profissional experiente. Entendemos que esse profissional, e não o paciente, é quem determina a posição final, visando eliminar qualquer tendência do paciente em manter a cabeça em posição não natural (flexionada ou estendida). Além do que, os procedimentos efetuados para a obtenção das telerradiografias laterais em PNC não demonstraram ser mais difíceis ou mais demorados quando comparados aos do método convencional, já que ambos necessitam treinamento do técnico ou do clínico.

A Orientação Natural da Cabeça elimina em grande parte as alterações que poderiam ocorrer no registro da PNC, decorrentes de fatores externos, tais como a respiração bucal, a deficiência visual, e procedimentos ortodôntico-ortopédicos. O presente estudo se baseou na comprovação da eficácia da ONC para a realização da metodologia, além do fato de termos utilizado o espelho como referência externa, já que em pesquisas nas quais foram feitas comparações de métodos com e sem espelho para a orientação da cabeça, notouse que, quando foi utilizado, houve menor variação na posição da cabeça. Acreditamos, portanto, que tenham sido estes alguns dos motivos para a reprodutibilidade ser notavelmente alta, mesmo existindo uma variável (expansão rápida da maxila) entre as séries de radiografias.

Partindo do princípio que mesmo com a utilização de mecanoterapia para a expansão rápida da maxila, que segundo a literatura pode melhorar o padrão respiratório, aumentar a altura facial inferior e conseqüentemente promover alterações de posicionamento da cabeça que poderiam comprometer a reprodutibilidade do método de PNC, conseguimos comprovar por este estudo que seguindo cuidadosamente o protocolo para a obtenção de telerradiografia lateral descrito e sob orientação criteriosa de uma pessoa qualificada, o método é confiável e reproduzivel, principalmente quando é considerada a variabilidade inerente das linhas de referência cefalométricas intracranianas. 


\section{CONCLUSÕES}

Após a análise estatística dos dados e a discussão dos resultados obtidos, concluímos que, na comparação das fases pré e pós-ERM no presente estudo:

1) A cabeça não apresentou alteração estatisticamente significativa, tanto em relação à linha vertical verdadeira (SN.VER) quanto à coluna cervical (OPT.SN e CVT.SN).

2) A inclinação da coluna cervical não apresentou alteração significativa em relação à linha horizontal verdadeira (OPT.HOR e CVT.HOR).

3) A curvatura cervical não apresentou alteração significativa (OPT.CVT).

4) Não houve diferenças estatisticamente significativas entre os gêneros, quando comparados os momentos pré e pós-ERM, para as grandezas estudadas.

5) O método de obtenção de telerradiografias em Posição Natural da Cabeça mostrou ser confiável e reproduzível $\left(2,91^{\circ}\right)$, mesmo na presença de intervenção ortodôntica-ortopédica entre as séries de radiografias.

\title{
Cephalometric radiographic evaluation of the craniocervical position of patients oriented in natural head position before and after the rapid maxillary expansion
}

\begin{abstract}
The aim of this study was to evaluate the eventual alteration in the craniocervical position of patients, oriented in the Natural Head Position (NHP), after the rapid maxillary expansion (RME) procedure. We have used lateral radiographs, selected from the documentation file of the Preventive Orthodontics Clinic, School of Dentistry, University of São Paulo. Our sample consisted of 20 patients (7M:13F), with ages ranging from 5.08 up to 11.08 years (mean of 9,0 ). The cephalometric radiographs were obtained in the beginning of the treatment and immediately after the active phase of the maxilla expansion. All patients showed constrictions of the maxilla, and were treated with modified Biederman appliance. Craniocervical cephalometric analysis was carried out by evaluating the craniovertical (SN.VER), craniocervical (OPT.SN e CVT.SN), cervicohorizontal (OPT.HOR e CVT.HOR) and the cervical curve (OPT. CVT) angles. On the basis of the statistical analysis of the data, we conclude that the head position did not show significant alteration. Statistically significant differences were not detected between the sexes, when the phases pre and post RME were compared for the measurements studied. In the sample studied, the method of obtaining cephalometric radiographs in the Natural Head Orientation showed to be trustworthy and reproducible, even in the presence of orthodontic-orthopedic intervention between the series of radiographs.
\end{abstract}

Key words: Orthodontic. Natural head position. Cephalometric radiographs. Rapid maxillary expansion.

\section{REFERÊNCIAS}

1. ALMEIDA, G. de A.; CAPELOZZA FILHO, L.; TRINDADE JR., A. S. Expansão rápida da maxila: estudo cefalométrico prospectivo. Ortodontia, São Paulo, v. 32, n. 1, p. 45-56, jan./abr. 1999.

2. ANDRIGHETTO, A. R.; PAIVA, J. B. de; FANTINI, S. M. de Avaliação cefalométrica radiográfica da posição craniocervical antes e após a desprogramação neuromuscular em pacientes com maloclusão classe II de Angle. Ortodontia, São Paulo, v. 34, n. 3, p. 19-25, set./dez. 2001.

3. BIEDERMAN, W. A. A hygienic appliance for rapid expansion. J Pract Orthod, Hempstead, v. 2, no. 2, p. 67-70, Feb. 1968.

4. BISHARA, S. E.; STALEY, R. Maxillary expansion: clinical implications. Am J Orthod Dentofacial Orthop, St. Louis, v. 91, no. 1, p. 3-14, Jan. 1987.

5. BJERIN, R. A. A comparison between the Frankfurt horizontal and the sell turcica-nasion as reference plane in cephalometric analysis. Acta Odontol Scand, Stockolm, v. 15, no. 1, p. 1-12, 1957.
6. BROADBENT, B. H. A new $X$-ray technique and its application to orthodontia. Angle Orthod, Appleton, v. 2, p. 45-66, 1931.

7. COOKE, M. S.; WEI, S. H. Y. A summary five-factor cephlometric analysis based on natural head posture and the true horizontal. Am J Orthod Dentofacial Orthop, St. Louis, v. 93, no. 3, p. 213-223, Mar. 1988a.

8. COOKE, M. S.; WEI, S. H. Y.The reproducibility of natural head posture: a methodological study. Am J Orthod Dentofacial Orthop, St. Louis, v. 93, no. 4, p. 280-288, Apr. 1988b.

9. COOKE, M. S. Five-year reproducibility of natural head posture: a longitudinal study. Am J Orthod Dentofacial Orthop, St. Louis, v. 97, no. 6, p. 489-494, June 1990.

10. DOWNS, W. B. Analysis of dento-facial profile. Angle Orthod, Appleton, v.26, no. 4, p. 191-212, Oct. 1956.

11. FJELLVANG, H.; SOLOW, B. Craniocervical postural relations and craniofacial morphology in 30 blind subjects. Am J Orthod Den- 
tofacial Orthop, St. Louis, v. 90, no. 4, p. 327-334, Oct. 1986.

12. FLEISS, J. L. Design and analysis of clinical experiments. New York: John Wiley, 1986.

13. FOSTER, T. D.; HOWAT, A. P.; NAISH, P. J. Variation in cephalometric reference lines. $\mathbf{B r} \mathbf{J}$ Orthod, London, v. 8, no. 4, p. 183-187, Oct. 1981

14. GREENFIELD, B.; KRAUS, S.; LAWRENCE, E.; WOLF, S. L. The influence of cephalostatic ear rods on the positions of the head and neck during postural recordings. Am J Orthod Dentofacial Orthop, St. Louis, v. 95, no. 4, p. 312-318, Apr. 1989.

15. HAAS, A. J. Rapid expansion of the maxillary dental arch and nasal cavity by opening the midpalatal suture. Angle Orthod, Appleton, v. 31, no. 2, p. 73-90, Apr. 1961.

16. HALAZONETIS, D. J. Estimated natural head position and facial morphology. Am J Orthod Dentofacial Orthop, St. Louis, v. 121, no. 4, p. 364-368, Apr. 2002.

17. HARTGERINK, D. V.; VIG, P. S.; ABBOTT, D. W. The effect of rapid maxillary expansion on nasal airway resistance. Am J Orthod Dentofacial Orthop, St. Louis, v. 92, no. 5, p. 381-389, Nov. 1987.

18. HERSHEY, H. G.; STEWART, B. L.; WARREN, D. W. Changes in nasal airway resistance associated with rapid maxillary expansion. Am J Orthod, St. Louis, v. 69, no. 3, p. 274-284, Mar. 1976.

19. HOUSTON, W. J. B. The analysis of errors in orthodontic measurements. Am J Orthod, St. Louis, v. 83, no. 5, p. 382-390, May 1983.

20. HOUSTON, W. J. B. Bases for the analysis of cephlometric radiographs: intracranial reference structures or natural head position. Proc Finn Dent Soc, Helsinki, v. 87, no. 1, p. 43-49, 1991.

21. HUGGARE, J. A. V.; LAINE-ALAVA, T. Nasorespiratory function and head posture. Am J Orthod Dentofacial Orthop, Saint Louis, v. 112, no. 5, p. 507-511, Nov. 1997.

22. INTERLANDI, S. Ortodontia: bases para iniciação. 4. ed. São Paulo: Artes Médicas, 1999.

23. KAWAKAMI, R. Y.; HENRIQUES, J. F. C.; PINZAN, A.; FREITAS, M. R. de; JANSON, G. dos R. P. Comparação dos efeitos dento esqueléticos, produzidos por dois tipos de disjuntores palatinos, por meio de análise cefalométrica em norma lateral. Ortodontia, São Paulo, v. 32, n. 3, p. 8-27, set./dez. 1999.

24. LEITÃO, P.; NANDA, R. S. Relationship of natural head position to craniofacial morphology. Am J Orthod Dentofacial Orthop St. Louis, v. 117, no. 4, p. 406-417, Apr. 2000.

25. LUNDSTRÖM, F. Registration of natural head posture in children. Swed Dent J, Stockholm, v. 15, p. 147-152, 1982.

26. LUNSTRÖM, F.; LUNDSTRÖM, A.; LEBRET, L. M. L.; MOORREES, C. F. A. Natural head position and natural head orientation: basic considerations in cephalometric analysis and research. Eur J Orthod, London, v. 17, no. 2, p. 111-120, Apr. 1995.

27. LUNDSTRÖM, A.; LUNDSTRÖM, F. The Frankfort horizontal as a basis for cephalometric analysis. Am J Orthod Dentofacial Orthop, St. Louis, v. 107, no. 5, p. 537-540, May 1995.

28. LUSVARGHI, L. Identificando o respirador bucal. R Assoc Paul Cir Dent, São Paulo, v. 53, n. 4, p. 265-274, jul./ago. 1999.

29. MARCHIORO, E. M.; RIZZATTO, S. D.; ROITHMANN, R.; LUBIANCA, J. F.N. O efeito da expansão rápida da maxila na geometria e função nasal-relato de caso. Ortodontia Gaucha Porto Alegre, v. 1, n. 1, p. 3-7, abr. 1997.

30. MARTON, N.; MARTINS, N. dos S. Estudo comparativo das radiografias cefalométricas laterais em posição convencional e em postura natural da cabeça (postura preconizada por Rocabado) uma análise dos arcos dentários. Ortodontia, São Paulo, v. 34, n. 2, p. 73-79, maio/ago. 2001.

31. MIRALLES, R.; MOYA, H.; RAVERA, M. J.; SANTANDER, H.; ZÚÑIGA, C.; CAVAJAL, R.; YAZIGI, C. Increase of the vertical occlusal dimension by means of a removable orthodontic appliance and its effect on craniocervical relationships and position of the cervical spine in children. Cranio, Baltimore, v. 15, no. 3, p. 221-228, July 1997

32. MOORREES, C. F. A.; KEAN, M. R. Natural head position: a basic consideration in the interpretation of cephalometric radiographs. Am J Phys Anthropol, New York, v. 16, no. 2, p. 213-234, June 1958
33. MOORREES, C. F. A. Natural head position. In: JACOBSON, A.; CAUFIELD, P. W. Introduction to radiographic cephalometry. Philadelphia: Lea \& Febiger,1985. cap. 8, p. 84-89.

34. MOORREES, C. F. A. Natural head position - a revival. Am J Orhod Dentofacial Orthop, St. Louis, v. 105, no. 5, p. 512-513, May 1994.

35. NETER, J.; KUTNER, M. H.; NACHTSHEIM, C. J.; WASSERMAN, W. Applied linear statistical models. 4rd ed. Ilinois: Richard D. Irwing, 1996.

36. OZBEK, M. M.; KÖKLÜ, A. A extracranial versus intracranial references in individual cephalometric analysis. Br J Orthod, London, v. 21, no. 3, p. 259-263, Aug. 1994.

37. PAIVA, J. B. de; VIGORITO, J. W.; CASTRO, A. B. B. A. T. de. Estudo rinomanométrico da cavidade nasal em pacientes submetidos à expansão rápida da maxila. Ortodontia, São Paulo, v. 33, n. 1, p. 36-42, jan./abr. 2000

38. PENG, L.; COOKE, M. S. Fifteen-year reproducibility of natural head posture: a longitudinal study. Am J Orthod Dentofacial Orthop, St. Louis, v. 116, no. 1, p. 82-85, July 1999.

39. RICKETTS, R. M. Respiratory obstruction syndrome. Am J Orthod, St. Louis, v. 54, no. 7, p. 495-507, July 1968

40. RINK, J. F; VIG, P. S.; SHOWFETY, K. J. The influence of visual targets on natural head position. J Dent Res, Chicago, v. 62, p. 285, Mar. 1983.

41. RINO NETO, J.; PAIVA, J. B. de; FREIRE-MAIA, B. A. V.; MIASIRO JÚNIOR, $H_{\text {.; }}$ ATTIZZANI, M. F.; CRIVELLO JUNIOR, O. Avaliação da reprodutibilidade da posição natural da cabeça: um estudo radiográfico. Ortodontia, São Paulo, v. 35, n. 4, p. 55-68, out/dez. 2002.

42. SANDHAM, A. Repeatability of head posture recordings from lateral cephalometric radiographs. Br J Orthod, London, v. 15, no. 3, p. 157-162, Aug. 1988.

43. SCHWARZ, A. M. Positions of the head and malrelations of the jaws. Int J Orthod Oral Surg Radiog, St. Louis, v. 14, no. 1, p. 56-68, Jan. 1928.

44. SHOWFETY, K. J.; VIG, P. S.; MATTESON, S. A simple method for taking natural-head-position cephalograms. Am J Orthod, St. Louis, v. 83, no. 6, p. 495-500, June 1983.

45. SIERSBAEK-NIELSEN, S.; SOLOW, B. Intra- and inter-examiner variability in head posture recorded by dental auxiliaries. Am J Orthod Dentofacial Orthop, St. Louis, v. 82, no. 1, p. 50-57, July 1982.

46. SILVA FILHO, O. G. da; FREITAS, S. F. de; CAVASSAN, A. de O. Hábitos de sucção: elementos passíveis de intervenção. Estomatol Cult, Bauru, v. 16, n. 4, p. 61-71, out./dez. 1986.

47. SILVA FILHO, O. G. da; VILLAS BOAS, M. C. ; CAPELOZZA FILHO, L. Rapid maxillary expansion in the primary and mixed dentitions. A cephalometric evaluations. Am J Orthod Dentofacial Orthop, St. Louis, v. 100, no. 2, p. 171- 179, Aug. 1991.

48. SINGER, J. M.; ANDRADE, D. F. Analysis of longitudinal data. In: SEM, P. K.; RAO, C. R. (Ed.). Handbook of Statistics. Amesterdam: North Holland, 2000. v. 18, p. 115-160.

49. SOLOW, B.; TALLGREN, A. A natural head position in standing subjects. Acta Odontol Scand, Stockolm, v. 29, no. 5, p. 591607, Nov. 1971

50. SOLOW, B.; TALLGREN, A. Head posture and craniofacial morphology. Am J Phys Anthropol, New York, v. 44, no. 3, p. 417436, May 1976.

51. SOLOW, B.; SIERSBAEK-NIELSEN, S.; GREVE, E. Airway adequacy, head posture, and craniofacial morphology. Am J Orthod Dentofacial Orthop, St. Louis, v. 86, no. 3, p. 214-223, Sept. 1984.

52. SOLOW, B.; SONNESEN, L. Head posture and malocclusions. Eur J Orthod, London, v. 20, no. 6, p. 685-693, Dec. 1998.

53. SUBTELNY, J. D. The significance of adenoid tissue in orthodontia. Angle Orthod, Appleton, v. 24, no. 2, p. 59-64, Apr. 1954

54. TIMMS, D. J. The effect of rapid maxillary expansion on nasal airway resistance. Br J Orthod, London, v. 13, no. 4, p. 221-228, Oct. 1986.

55. TIMMS, D. J. Rapid maxillary expansion in the treatment of nocturnal enuresis. Angle Orthod, Appleton, v. 60, no. 3, p. 229234, Fall 1990 
56. TNG, T. T. H.; CHAN, T. C. K.; COOKE, M. S.; HÄGG, U. Effect of head posture on cephalometric sagittal angular measures. Am J Orthod Dentofacial Orthop, St. Louis, v. 104, no. 4, p. 337341, Oct. 1993.

57. TOURNE, L. P. M.; SCHWEIGER, J. Immediate postural responses to total nasal obstruction. Am J Orthod Dentofacial Orthop, St. Louis, v. 110, no. 6, p. 606-611, Dec. 1996.

58. URBANOWICZ, M. Alteration of vertical dimension and its effect on head and neck posture. Cranio, Baltimore, v. 9, no. 2, p. 174-179, Apr. 1991.

59. VIAZIS, A. D. Natural head position In:__. Atlas of orthodontics: principles and clinical applications. Philadelphia: W. B. Saunders, 1993. cap. 1, p. 41-43.

60. VIG, P. S.; SHOWFETY, K. J.; PHILLIPS, C. Experimental manipu- lation of head posture. Am J Orthod Dentofacial Orthop, St. Louis, v. 77, no. 3, p. 258-268, Mar. 1980.

61. VIGORITO, J. W. Ortodontia: clínica preventiva. 2. ed. São Paulo: Artes Médicas, 1986.

62. WERTZ, R. A. Changes in nasal airflow incident to rapid maxillary expansion. Angle Orthod, Appleton, v. 33, no. 1, p. 1-11, Jan. 1968.

63. WEBER, Z. J.; PRESTON, C. B.; WRIGHT, P. G. Resistance to nasal airflow related to changes in head posture. Am J Orthod Dentofacial Orthop, St. Louis, v. 80, no. 5, p. 536-545, Nov. 1981.

64. WOODSIDE, D. G.; LINDER-ARONSON, S.; LUNDSTRÖM, A.; McWILLIAN, J. Mandibular and maxillary growth after changed mode of breathing. Am J Orthod Dentofacial Orthop, St. Louis, v. 100 , no. 1 , p. $1-18$, Jan. 1991
Endereço para correspondência

Belini Augusto Villalba Freire-Maia

Faculdade de Odontologia da Universidade de São Paulo

Disciplina de Ortodontia

Av. Prof. Lineu Prestes, 2227 - Cidade Universitária - São Paulo/SP

CEP: 05508-900

E-mail: belini@usp.br 